\title{
ERROR ESTIMATION FOR QUADRATURE FORMULAS BASED ON EQUALLY SPACED NODES
}

\author{
K. PLUKAS and D. PLUKIENE் \\ Kaunas University of Technology \\ Studentu 50-407, 3031 Kaunas, Lithuania \\ E-mail: Kostas.Plukas@ktu. It \\ Received October 15, 2005; revised April 04, 2006; published online September 15, 2006
}

\begin{abstract}
The error estimation for quadrature formulas based on equally spaced nodes is discussed in this paper. The error estimates use embedded formulas and they are obtained for Newton-Cotes and Hermitian quadrature formulas. The coefficients of these formulas and error estimates are presented. The locally adaptive integration procedures implementing the truncation error estimation method proposed in this paper were developed in MATLAB and results of appropriate comparative tests are presented.

Key words: numerical integration, quadrature formula, error estimate, locally adaptive procedure
\end{abstract}

\section{Introduction}

Numerical approximation of integrals $I f=\int_{a}^{b} f(x) d x$ is one of the most frequently used numerical procedure. Numerous techniques that are available for analytical evaluation of integrals are inadequate for many problems that arise in the real physical world. Consequently the value of the definite integral must be approximated by some numerical methods.

Most numerical integration methods involve constructing the interpolating polynomial of $f(x)$ and then integrating this polynomial in order to obtain an approximation to the integral of $f(x)$. All interpolatory quadrature formulas are uniquely characterized by the choice of their nodes $x_{1}, x_{2}, \ldots, x_{n}$. The most important sets of interpolatory quadrature formulas are the following.

Closed Newton-Cotes formulas. Closed Newton-Cotes formulas $Q_{n}^{N}, n \geqslant 2$ are interpolatory formulas based on equidistant nodes

$$
x_{i}=a+(i-1) h, \quad i=1,2, \ldots, n, h=\frac{b-a}{n-1}
$$


and can be presented as

$$
Q_{n}^{N} f=h \sum_{i=1}^{n} w_{i} y_{i}
$$

where $w_{i}$ are the coefficients called the weights, $y_{i}=f\left(x_{i}\right)$ are the values of the integrand evaluated at the nodes $x_{i}$, and $h$ is an integration step.

Closed Hermitian quadrature formulas. Closed Hermitian quadrature formulas $Q_{n}^{H}$ are also based on interpolation functions constructed on equidistant nodes and can be presented as

$$
Q_{n}^{H} f=h \sum_{i=1}^{n} w_{i}^{H} y_{i}+h^{2} \sum_{i=1}^{n} w_{i}^{H^{*}} y_{i}{ }^{\prime},
$$

where $w_{i}^{H}, w_{i}^{H^{*}}$ are coefficients called the weights, $y_{i}=f\left(x_{i}\right)$ and $y_{i}^{\prime}=f^{\prime}\left(x_{i}\right)$ are the values of the integrand and its derivative evaluated at the nodes $x_{i}$.

Gaussian quadrature formulas. Gauss-Legendre quadrature formulas $Q_{n}^{G}$ are also based on interpolation functions constructed on the nodes chosen as zeros of orthogonal Legendre polynomials and can be presented as

$$
Q_{n}^{G} f=h \sum_{i=1}^{n} w_{i}^{G} y_{i}
$$

where $w_{i}^{G}$ are coefficients called the weights.

Definition 1. [2]. A quadrature formula $Q_{n}$ has degree $d$ if it integrates exactly all polynomials of degree $\leq d$ and fails to integrate exactly $f(x)=$ $x^{d+1}$.

Definition 2. The truncation error for the quadrature formula is the difference between the numerical and analytical (exact) values of the integral.

The truncation error for the quadrature formulas (1.1)-(1.3) is given in the form

$$
C h^{\alpha} f^{(\beta)}(\xi)
$$

where $C$ is a constant, $\alpha$ is an integer dependent on the degree of quadrature formula, $\beta$ is an integer indicating the order of the derivative, and $\xi$ is some point in the interval $(a, b)$.

Suppose that we want to approximate integral $\int_{a}^{b} f(x) d x$ to within a specified tolerance $\varepsilon>0$. For solving this problem we need: a quadrature formula, an integration strategy, and a formula for estimation of the truncation error.

An important problem associated with automatic numerical integration problem is that of error estimation. It is difficult to compute error estimates that are both reliable and accurate. An obvious way to obtain an error estimate is to compare a pair of integration formulas $Q_{n 1}$ and $Q_{n 2}$. The assumption that $Q_{n 2}$ returns significantly better results than $Q_{n 1}$ implies that error estimate formula 


$$
\Delta=\left|Q_{n 1} f-Q_{n 2} f\right|
$$

gives a sufficiently accurate estimate of the truncation error for a large class of integrands. A necessary condition in this technique is the assumption that the degree of accuracy $Q_{n 2}$ is larger than $Q_{n 1}$.

A simple way to obtain two different estimates $Q_{n 1}$ and $Q_{n 2}$ for $I f$ is to use compound rules. Integration formula $Q_{n}$ is applied first to $[a, b]$ and then to the two subintervals $[a,(a+b) / 2]$ and $[(a+b) / 2, b]$. Adding up the results of both subintervals leads to the formula $Q_{2 n}$ which is more accurate than $Q_{n}$. If $f$ is a sufficiently smooth function and $Q_{n}$ has the degree $p$ then formula

$$
E=\left|\frac{Q_{2 n} f-Q_{n} f}{2^{p}-1}\right|
$$

may be used as an error estimation formula.

The main drawback of formula (1.6) is necessity to approximate definite integral twice. Therefore for minimization of computation costs the estimates $Q_{n 1}$ and $Q_{n 2}$ are calculated using embedded formulas. Suppose the $n+1$ nodes are fixed and a unique interpolatory quadrature formula $Q_{n 2}=Q_{n+1}$ of degree $d$ is constructed. The formula $Q_{n 1}$ may be constructed removing one or some points from the initial set of nodes checking that the interpolatory rule based on the remaining nodes actually has lower degree of accuracy than the formula $Q_{n 2}$.

Definition 3. [2]. A rule

$$
N f=\sum_{i=1}^{n} u_{i} f\left(x_{i}\right)
$$

is a null rule if it has at least one nonzero weight, and in addition

$$
\sum_{i=1}^{n} u_{i}=0
$$

A null rule is said to have degree $d$ if it integrates to zero all polynomials of degree $\leq d$ and fails to do so with $f(x)=x^{d+1}$. A null rule $N$ and interpolatory rule $Q$, based on the same set of points, are equally strong if the null rule is scaled such that $\|N\|_{2}=\|Q\|_{2}$, where

$$
\|N\|_{2}=\left(\sum_{i=1}^{n} u_{i}^{2}\right)^{1 / 2}, \quad\|Q\|_{2}=\left(\sum_{i=1}^{n} w_{i}^{2}\right)^{1 / 2}
$$

and $w_{i}$ are the coefficients of rule $Q$. The term null rule was first used in 1965 by J. N. Lyness [15]. The general theory of null rules was presented in 1991 by J. Berntsen and T.O. Espelid in [2].

Gauss formulas have one severe drawback: two arbitrary Gauss formulas $Q_{n 1}^{G}$ and $Q_{n 2}^{G}$ with $n_{2}>n_{1}$ do not have any identical nodes (except, 
perhaps, for the interval center). The usual procedure for obtaining a practical error estimate by formula (1.5) requires too many integrand values and therefore too much computational effort. This disadvantage was overcome by using a seemingly obvious method proposed by A. S. Kronrod in 1965 [17]. Suppose that $n$ nodes $x_{1}, x_{2}, \ldots, x_{n}$ are the abscissas of $Q_{n 1}^{G}$, then formula $Q_{n 2}^{G}$ with $(2 n+1)$ nodes is constructed in such a way that its degree of accuracy is the highest possible, i.e. new nodes are contained in the intervals $\left(a, x_{1}\right),\left(x_{1}, x_{2}\right), \ldots,\left(x_{n}, b\right)$.

Since the first automatic algorithm for the numerical calculation of definite integrals was given by McKeeman [16] in 1963, many new algorithms, including non-adaptive, adaptive (locally and globally), and double adaptive (locally and globally), have been developed [3, 4, 5, 6, 7, 8, 9, 13, 14].

Recently Gander and Gautschi [9] published a paper describing two new adaptive quadrature Matlab codes adaptsim and adaptlob based on Simpson's and the four-point Gauss-Lobatto rules respectively. Espelid in [3] discussed null rules based on divided differences and constructed error estimator using sequences of null rules with the intention to increase the reliability for both adaptsim and adaptlob codes. In addition two new Matlab codes coteda and coteglob are developed, they use a locally and a globally double adaptive strategy respectively. Both algorithms use sequences of null rules in their local error estimations. These new codes make use of both the five-point closed Newton-Cotes rule and the nine-point closed Newton-Cotes rule.

The aims of this paper are:

1. To compute the null rules with the smallest truncation error for NewtonCotes and Hermitian quadrature formulas.

2. To develop and to test adaptive Matlab procedures based on the constructed null rules and the nine-point closed Newton-Cotes and the fourpoint closed Hermitian quadrature formulas.

3. To compare numerical results with Gander-Gautschi and Espelid results from the well known test kit given in $[3,9]$.

\section{Truncation Error Evaluation}

Suppose that quadrature $Q$ is based on nodes $x_{0}=a, x_{1}, x_{2}, \ldots, x_{n}=b$ and it has the degree $d$ and quadrature $Q_{1}$ is based on the same nodes except of nodes $x_{k_{1}}, \ldots, x_{k_{q}}, k_{i} \in\{1,2, \ldots, n-1\}, i=\overline{1, q}, q \geq 1$ and it has degree $d_{1} \leq(d-q)$. Usually, the nodes $x_{k_{i}}, i=\overline{1, q}$ are chosen in such a way that the quadrature formula $Q_{1}$ would have the smallest theoretical truncation error, i.e. that the absolute value of the constant $C$ in the truncation error formula (1.4) would be the smallest.

Suppose that $\Delta$ and $\Delta_{1}$ are the values of truncation error for formulas $Q$ and $Q_{1}$ respectively. Thus,

$$
I f=Q f+\Delta=Q_{1} f+\Delta_{1} .
$$

Equivalently, (2.1) can be rewritten as 


$$
\delta=\left|Q f-Q_{1} f\right|=\left|\Delta_{1}-\Delta\right| .
$$

It is obvious that formula (2.2) is the null rule of degree $d_{0}=\min \left(d, d_{1}\right)$. It implies that formula (2.2) can be used for local error estimation.

We may expect that error estimate formula (2.2) on the narrow integration interval will be exact enough because

$$
\left|f(x)-P_{n}(x)\right| \approx\left|P_{n+1}(x)-P_{n}(x)\right|,
$$

when interpolating step is sufficiently small, where $P_{n}(x)$ and $P_{n+1}(x)$ are interpolating polynomials for $f(x)$ [1]. This statement can be proved by using the Taylor series in the interval $\left[x_{j}, x_{j+1}\right]$.

Formula (2.2) for the closed Newton-Cotes rules can be written as

$$
\delta=\left|Q f-Q_{1} f\right|=\left|h\left(w_{1}^{\delta} y_{1}+w_{2}^{\delta} y_{1}+\ldots+w_{n}^{\delta} y_{n}\right)\right|,
$$

where $w_{i}^{\delta}=w_{i}^{Q}-w_{i}^{Q_{1}}, i=0, \ldots, n$, and the coefficients $w_{i}^{Q}$ and $w_{i}^{Q_{i}}$ are the weights in formula (1.1) for the quadrature formulas $Q$ and $Q_{1}$ respectively.

In a similar way formula (2.2) for closed Hermitian rule can be written as

$$
\delta=\left|h\left(w_{1}^{\delta} y_{1}+w_{2}^{\delta} y_{1}+\ldots+w_{n}^{\delta} y_{n}\right)+h^{2}\left(w_{1}^{\delta^{*}} y_{1}^{\prime}+w_{2}^{\delta^{*}} y_{2}^{\prime}+\ldots+w_{n}^{\delta^{*}} y_{n}^{\prime}\right)\right| .
$$

\section{The Error Estimation for Newton-Cotes Quadrature Formulas}

Suppose that the closed Newton-Cotes quadrature formula $Q$ is based on equally spaced nodes $x_{i}, i=\overline{0, n}$ and it has the degree $n$. In this section we will discuss a procedure for construction of a closed interpolatory quadrature formula $Q_{1}$, which is based on the same nodes $x_{i}$ except of one node $x_{k}, k \in$ $\{1,2, \ldots, n-1\}$ and the truncation error of which is the smallest possible.

The construction of quadrature $Q_{1}$ consists of the following steps.

1. Substitute the integrand function $f(x)$ with the Lagrange interpolating polynomial $P_{n-1}(x)$ that agrees with $f(x)$ at nodes $x_{i}, i=\overline{0, n}, i \neq k, k_{i} \in$ $\{1,2, \ldots, n-1\}$.

2. Write the resulting approximation of the integral

$$
\int_{a}^{b} f(x) d x \approx Q_{1} f=\int_{a}^{b} P_{n-1}(x) d x=h \sum_{i=0, i \neq k}^{n} w_{i}^{Q_{1}} y_{i} .
$$

3. For each $k=1,2, \ldots, n-1$ evaluate the truncation errors of formula (3.1).

4. Choose the smallest theoretical truncation error. Let $x_{k}$ be a node for which this error is received.

5. Calculate the coefficients $w_{i}^{Q_{i}}$ in formula (3.1). 
The theoretical truncation error $\Delta_{1}$ for quadrature $Q_{1}$ is calculated using the error formula of the interpolating polynomial $P_{n-1}(x)$ and the Intermediate Value Theorem. It can be written as

$$
\Delta_{1}=\frac{f^{(n)}(\xi)}{n !} \int_{x_{0}=a}^{x_{n}=b}\left(x-x_{0}\right)\left(x-x_{1}\right) \ldots\left(x-x_{k-1}\right)\left(x-x_{k+1}\right) \ldots\left(x-x_{n}\right) d x,
$$

where $\xi$ is some point in the interval $(a, b)$. Using substitution $t=\frac{x-a}{h}-l$, where $h=\frac{b-a}{n}, l=\frac{n}{2}$, formula (3.2) can be written as

$\Delta_{1}=\frac{f^{(n)}(\xi)}{n !} h^{n+1} \int_{-l}^{l}(t+l)(t+l-1) \ldots(t+l-k+1)(t+l-k-1) \ldots(t-l) d t$.

Formula (3.3) is more convenient for calculation, because it does not depend on the actual values of $x_{i}$.

The coefficients in formula (3.1) can be computed using the method of undetermined coefficients. Formula (3.1) must be exact for polynomials of degree $\leq(n-1)$. In this case, we need to solve the following system of linear equations

$$
\left\{\begin{array}{l}
w_{0}^{Q_{1}} \varphi_{j}\left(l_{0}\right)+w_{1}^{Q_{1}} \varphi_{j}\left(l_{1}\right)+\ldots+w_{k-1}^{Q_{1}} \varphi_{j}\left(l_{k-1}\right) \\
\quad+w_{k+1}^{Q_{1}} \varphi_{j}\left(l_{k+1}\right)+\ldots+w_{n}^{Q_{1}} \varphi_{j}\left(l_{n}\right)=m_{j}, \quad j=\overline{0, n-1},
\end{array}\right.
$$

where $\varphi_{j}\left(l_{i}\right)=(-l+i)^{j}, m_{j}=\int_{-l}^{l} x^{j} d x$, for all $i=0,1,2, \ldots, k-1, k+1, \ldots, n$, and $j=\overline{0, n-1}$.

Table 1 shows coefficients of the closed Newton-Cotes quadrature formulas, closed interpolatory quadrature formulas $Q_{1}$ and error estimation formula

$$
\Delta=c_{\Delta}(\tilde{p}) \frac{f^{(\tilde{p}-1)}(\xi)}{(\tilde{p}-1) !} h^{t i l d e p} .
$$

The table is divided into several parts, each of which consists of the three rows. The coefficients of the closed Newton-Cotes quadrature formula (quadrature $Q$ ) of the degree $p$ are put in the first row of each part. The calculated coefficients of the quadrature $Q_{l}$ with the smallest truncation error and of the degree $(p-2)$ are given in the second row, and the coefficients of the truncation error formula (2.3) are listed in the third row (denoted " $\delta_{n}$ "). These coefficients give the difference between coefficients in the first and in the second rows.

The column " $n$ " represents the number of equally sized subintervals into which integration interval is divided. The column " $p$ " gives the degree of quadrature formulas, and the column " $c_{\Delta}$ " displays theoretical formulas for evaluation of the truncation error. For example, if $n=4$, then the truncation 
Table 1. The coefficients of the symmetric embedded Newton-Cotes formulas and truncation error estimation formulas.

\begin{tabular}{|c|c|c|c|c|c|c|c|c|c|}
\hline$n$ & $p$ & $w_{0}$ & $w_{l}$ & $w_{2}$ & $w_{3}$ & $w_{4}$ & $w_{5}$ & $c_{\Delta}$ & $\tilde{p}$ \\
\hline \multirow{3}{*}{2} & 2 & $\frac{1}{3}$ & $\frac{4}{3}$ & $\frac{1}{3}$ & & & & $\frac{-4}{15}$ & 5 \\
\hline & 1 & 1 & 0 & 1 & & & & $\frac{-4}{3}$ & 3 \\
\hline & $\delta_{2}$ & $\frac{1}{3}$ & $\frac{4}{3}$ & $\frac{1}{3}$ & & & & & \\
\hline \multirow{3}{*}{4} & 4 & $\frac{14}{45}$ & $\frac{64}{45}$ & $\frac{24}{45}$ & $\frac{64}{45}$ & $\frac{14}{45}$ & & $\frac{-128}{21}$ & 7 \\
\hline & 3 & $\frac{2}{9}$ & $\frac{16}{9}$ & 0 & $\frac{16}{9}$ & $\frac{2}{9}$ & & $\frac{32}{15}$ & 5 \\
\hline & $\delta_{4}$ & $\frac{4}{45}$ & $\frac{-16}{45}$ & $\frac{24}{45}$ & $\frac{-16}{45}$ & $\frac{4}{45}$ & & & \\
\hline \multirow{3}{*}{6} & 6 & $\frac{41}{140}$ & $\frac{216}{140}$ & $\frac{27}{140}$ & $\frac{272}{140}$ & $\frac{27}{140}$ & $\frac{216}{140}$ & $\frac{-1296}{5}$ & 9 \\
\hline & 5 & $\frac{14}{50}$ & $\frac{81}{50}$ & 0 & $\frac{110}{50}$ & 0 & $\frac{81}{50}$ & $\frac{324}{35}$ & 7 \\
\hline & $\delta_{6}$ & $\frac{9}{700}$ & $\frac{-54}{700}$ & $\frac{135}{700}$ & $\frac{-180}{700}$ & $\frac{135}{700}$ & $\frac{54}{700}$ & & \\
\hline \multirow{3}{*}{8} & 8 & $\frac{3956}{14175}$ & $\frac{23552}{14175}$ & $\frac{-3712}{14175}$ & $\frac{41984}{14175}$ & $\frac{-18160}{14175}$ & $\frac{41984}{14175}$ & $\frac{-606208}{33}$ & 11 \\
\hline & 7 & $\frac{1908}{6615}$ & $\frac{10496}{6615}$ & 0 & $\frac{16128}{6615}$ & $\frac{-4144}{6615}$ & $\frac{16128}{6615}$ & $\frac{-118784}{315}$ & 9 \\
\hline & $\delta_{8}$ & $\frac{-928}{99225}$ & $\frac{7424}{99225}$ & $\frac{-25984}{99225}$ & $\frac{51968}{99225}$ & $\frac{-64960}{99225}$ & $\frac{51968}{99225}$ & & \\
\hline \multirow{3}{*}{10} & 10 & $\frac{80335}{299376}$ & $\frac{531500}{299376}$ & $\frac{-242625}{299376}$ & $\frac{1362000}{299376}$ & $\frac{-1302750}{299376}$ & $\frac{2136840}{299376}$ & $\frac{-53854 \cdot 10^{4}}{273}$ & 13 \\
\hline & 9 & $\frac{11690}{40824}$ & $\frac{65125}{40824}$ & 0 & $\frac{97500}{40824}$ & $\frac{-23250}{40824}$ & $\frac{106110}{40824}$ & $\frac{-647 \cdot 10^{4}}{99}$ & 11 \\
\hline & $\delta_{10}$ & $\frac{-16175}{898128}$ & $\frac{16175}{898128}$ & $\frac{-727875}{898128}$ & $\frac{1941000}{898128}$ & $\frac{-3396750}{898128}$ & $\frac{4076100}{898128}$ & & \\
\hline
\end{tabular}

error estimate formula (2.3) for the five point closed Newton-Cotes quadrature is presented as

$$
\delta_{4}=\frac{4}{45}\left|h\left(y_{0}-4 y_{1}+6 y_{2}-4 y_{3}+y_{4}\right)\right| .
$$

Remark 1. If $n=2$ or 4 , then we can use the composite rule for estimation of the truncation error: the composite trapezoid rule for $n=2$ and the composite Simpson's rule for $n=4$. In these cases we will get four times less the truncation error values than the corresponding values reported in Table 1.

Remark 2. Table 2 shows the ratio between the absolute values of the theoretical truncation errors of error estimation formulas $\delta_{n}\left(x_{k}\right)$. Here $\delta_{n}\left(x_{k}\right)$ denotes the theoretical error estimate for the $n$ degree quadrature formula based on equally spaced nodes $x_{0}, x_{1}, \ldots, x_{k-1}, x_{k+1}, \ldots, x_{n}$. Values $\left|\delta_{n}\left(x_{k}\right)\right|$ and $\left|\delta_{n}\left(x_{n-k}\right)\right|$ are equal.

It follows from Table 2 that optimal error estimator is obtained for $\delta_{n}\left(x_{2}\right)$. This fact can be explained in the way that for the even integer $n$ the value of integral $\int_{x_{0}=a}^{x_{n}=b}\left(x-x_{0}\right)\left(x-x_{1}\right) \ldots\left(x-x_{k-1}\right)\left(x-x_{k+1}\right) \ldots\left(x-x_{n}\right) d x$ has the smallest value if $x_{k}=x_{2}$. It based on the properties of polynomial ( $x-$ $\left.x_{0}\right)\left(x-x_{1}\right) \ldots\left(x-x_{k-1}\right)\left(x-x_{k+1}\right) \ldots\left(x-x_{n}\right)[12]$. 
Table 2. The values of the ratio $\left|\frac{\delta_{n}\left(x_{k}\right)}{\delta_{n}\left(x_{2}\right)}\right|$.

\begin{tabular}{cccccc}
\hline$n$ & $x_{1}$ & $x_{2}$ & $x_{3}$ & $x_{4}$ & $x_{5}$ \\
\hline 4 & 4 & 1 & 4 & & \\
6 & 20 & 1 & $\frac{68}{9}$ & 1 & 20 \\
8 & $\frac{644}{29}$ & 1 & $\frac{164}{29}$ & $\frac{227}{116}$ & $\frac{164}{29}$ \\
10 & $\frac{6378}{647}$ & 1 & $\frac{1362}{647}$ & $\frac{5211}{4529}$ & $\frac{35614}{23645}$ \\
\hline
\end{tabular}

\section{The Truncation Error Estimation for Hermitian Quadrature formulas}

The construction of the $(2 n+1)$-th order Hermitian quadrature formula $Q$, which is based on equidistant nodes, consists of the following steps.

1. Divide the given integration interval $[a, b]$ into equally sized subintervals by nodes $x_{i}=x_{0}+i h, i=\overline{0, n}$, where $x_{0}=a, h=(b-a) / n, x_{n}=b$.

2. Substitute integrand function $f(x)$ with the Hermite interpolating polynomial $H(x)$ obeying the following equalities:

$$
H\left(x_{i}\right)=y_{i}, \quad H^{\prime}\left(x_{i}\right)=y_{i}^{\prime}, \quad y_{i}=f\left(x_{i}\right), \quad y_{i}^{\prime}=f^{\prime}\left(x_{i}\right), \quad i=\overline{0, n} .
$$

3. Write the resulting approximation in the form

$$
\begin{aligned}
& \int_{a}^{b} f(x) d x \approx \int_{a}^{b} H(x) d x=h(\left.w_{0} y_{0}+w_{1} y_{1}+\cdots+w_{n} y_{n}\right) \\
&+h^{2}\left(w_{0}^{*} y_{0}^{\prime}+w_{1}^{*} y_{1}^{\prime}+\cdots+w_{n}^{*} y_{n}^{\prime}\right) .
\end{aligned}
$$

4. Calculate the coefficients $w_{i}$ and $w_{i}^{*}$.

The coefficients in formula (4.1) are computed using the undetermined coefficients method. Hence, like for the Newton-Cotes formulas, we need to solve the following system of linear equations

$$
\left\{\begin{array}{l}
w_{0} \varphi_{j}\left(l_{0}\right)+w_{1} \varphi_{j}\left(l_{1}\right)+\cdots+w_{n} \varphi_{j}\left(l_{n}\right) \\
\quad+w_{0}^{*} \varphi_{j}^{\prime}\left(l_{0}\right)+w_{1}^{*} \varphi_{j}^{\prime}\left(l_{1}\right)+\cdots+w_{n}^{*} \varphi_{j}^{\prime}\left(l_{n}\right)=m_{j}, \quad j=\overline{0,2 n+1},
\end{array}\right.
$$

where

$$
\begin{aligned}
& \varphi_{j}\left(l_{i}\right)=(-[n / 2]+i)^{j}, \quad \varphi_{j}^{\prime}\left(l_{i}\right)=j(-[n / 2]+i)^{j-1}, \quad i=\overline{0, n}, \\
& m_{j}=\int_{-[n / 2]}^{n-[n / 2]} x^{j} d x, j=\overline{0,2 n+1} .
\end{aligned}
$$


The theoretical truncation error $\Delta$ for Hermitian quadrature $Q$ can be computed using the truncation error formula of the Hermitian interpolating polynomial and the Intermediate Value Theorem. This formula like (3.3) can be written as follows

$$
\Delta=\frac{f^{(2 n+2)}(\xi)}{(2 n+2) !} h^{2 n+3} \int_{0}^{n} \prod_{l=0}^{n}(t-l)^{2} d t
$$

where $t=\frac{x-a}{h}, h=\frac{b-a}{n}$.

Suppose that Hermitian quadrature formula $Q$ is based on equally spaced nodes $x_{i},=\overline{0, n}$ and it has degree $p=2 n+1$. Then Hermitian quadrature formula $Q_{1}$ of degree $(p-q)$ for integer $q>0$ is obtained by the following steps.

1. Substitute integrand function $f(x)$ with the Hermite interpolating polynomial $H_{1}(x)$ obeying the following equations

$$
H_{1}(x)=y_{i}, \quad i=\overline{0, n}, \quad H_{1}^{\prime}(x)=y_{i}^{\prime}, \quad i=\overline{0, n}, i \neq k_{1}, k_{2}, \ldots, k_{q},
$$

where $x_{i}, i=\overline{0, n}$ are the nodes of the quadrature $Q$. The nodes

$$
x_{k_{i}} \in\left\{x_{0}, x_{1}, \ldots, x_{n}\right\}, \quad i=\overline{1, q}
$$

are chosen in such a way that the theoretical truncation error for quadrature $Q_{1}$ would be the smallest.

2. Write the resulting approximation in the form

$$
\int_{a}^{b} f(x) d x \approx \int_{a}^{b} H_{1}(x) d x=h \sum_{i=0}^{n} w_{i} y_{i}+h^{2} \sum_{i=0}^{n} w_{i}^{*} y_{i}^{\prime},
$$

where $w_{k_{i}}^{*}=0, i=\overline{1, q}$.

3. Calculate the coefficients $w_{i}$ and $w_{i}^{*}$.

The coefficients in (4.4) are computed by using the undetermined coefficients method. Formula (4.4) has degree $p_{1} \leq(2 n+1-q)$.

The theoretical truncation error $\Delta_{1}$ for Hermitian quadrature $Q_{1}$ is calculated using the error formula of the Hermite interpolating polynomial and the Intermediate Value Theorem. This formula like (4.3) can be written as follows

$$
\Delta_{1}=\frac{f^{(2 n+2-q)}(\xi)}{(2 n+2-q) !} h^{2 n+3-q} \int_{0}^{n} \prod_{l=0}^{n}(t-l)^{s} d t,
$$

where $t=\frac{x-a}{h}, h=\frac{b-a}{n}$ and if $l=k_{i}, i=\overline{1, q}$ then $s=1$ else $s=2$.

Tables 3 and 4 show calculated coefficients of the Hermitian quadrature formulas $Q$ and $Q_{1}$. The structure of both tables is the same as that of Table 1 .

Tables 5 and 6 show coefficients of the truncation error formulas of Hermitian quadrature. 
Table 3. The coefficients of the embedded Hermitian quadrature formulas.

\begin{tabular}{|c|c|c|c|c|c|c|c|c|}
\hline$n$ & $\delta$ & $w_{0}$ & $w_{1}$ & $w_{2}$ & $w_{3}$ & $w_{4}$ & $w_{5}$ & $w_{6}$ \\
\hline \multirow[b]{2}{*}{1} & 3 & $\frac{1}{2}$ & $\frac{1}{2}$ & & & & & \\
\hline & 2 & $\frac{1}{3}$ & $\frac{2}{3}$ & & & & & \\
\hline \multirow[b]{2}{*}{2} & 5 & $\frac{7}{15}$ & $\frac{16}{15}$ & $\frac{7}{15}$ & & & & \\
\hline & 4 & $\frac{4}{15}$ & $\frac{16}{15}$ & $\frac{10}{15}$ & & & & \\
\hline \multirow[b]{2}{*}{3} & 7 & $\frac{93}{224}$ & $\frac{243}{224}$ & $\frac{243}{224}$ & $\frac{93}{224}$ & & & \\
\hline & 5 & $\frac{39}{80}$ & $\frac{81}{90}$ & $\frac{81}{90}$ & $\frac{39}{80}$ & & & \\
\hline \multirow{2}{*}{4} & 9 & $\frac{3202}{8505}$ & $\frac{8192}{8505}$ & $\frac{11232}{8505}$ & $\frac{8192}{8505}$ & $\frac{3202}{8505}$ & & \\
\hline & 7 & $\frac{434}{945}$ & $\frac{1024}{945}$ & $\frac{864}{945}$ & $\frac{1024}{945}$ & $\frac{434}{945}$ & & \\
\hline \multirow[b]{2}{*}{5} & 11 & $\frac{319085}{912384}$ & $\frac{691875}{912384}$ & $\frac{1270000}{912384}$ & $\frac{1270000}{912384}$ & $\frac{691875}{912384}$ & $\frac{319085}{912384}$ & \\
\hline & 9 & $\frac{175515}{435456}$ & $\frac{455625}{435456}$ & $\frac{457500}{435456}$ & $\frac{457500}{435456}$ & $\frac{455625}{435456}$ & $\frac{175515}{435456}$ & \\
\hline \multirow{2}{*}{6} & 13 & $\frac{3310219}{10010000}$ & $\frac{5014656}{10010000}$ & $\frac{11161125}{10010000}$ & $\frac{21088000}{10010000}$ & $\frac{1161125}{10010000}$ & $\frac{5014656}{10010000}$ & $\frac{3310219}{10010000}$ \\
\hline & 11 & $\frac{144341}{385000}$ & $\frac{377784}{385000}$ & $\frac{475875}{385000}$ & $\frac{314000}{385000}$ & $\frac{475875}{385000}$ & $\frac{377784}{385000}$ & $\frac{144341}{385000}$ \\
\hline
\end{tabular}

Table 4. The coefficients of the embedded Hermitian quadrature formulas.

\begin{tabular}{|c|c|c|c|c|c|c|c|c|c|c|}
\hline$n$ & $\delta$ & $w_{0}^{*}$ & $w_{1}^{*}$ & $w_{2}^{*}$ & $w_{3}^{*}$ & $w_{4}^{*}$ & $w_{5}^{*}$ & $w_{6}^{*}$ & $c_{\Delta}$ & $\tilde{p}$ \\
\hline \multirow[b]{2}{*}{1} & 3 & $\frac{1}{12}$ & $\frac{-1}{12}$ & & & & & & $\frac{1}{30}$ & 5 \\
\hline & 2 & 0 & $\frac{-1}{6}$ & & & & & & $\frac{1}{12}$ & 4 \\
\hline \multirow[b]{2}{*}{2} & 5 & $\frac{1}{15}$ & 0 & $\frac{-1}{15}$ & & & & & $\frac{16}{105}$ & 7 \\
\hline & 4 & 0 & $\frac{-4}{15}$ & $\frac{-2}{15}$ & & & & & $\frac{4}{15}$ & 6 \\
\hline \multirow{2}{*}{3} & 7 & $\frac{57}{1120}$ & $\frac{-81}{1120}$ & $\frac{81}{1120}$ & $\frac{-57}{1120}$ & & & & $\frac{81}{70}$ & 9 \\
\hline & 5 & $\frac{3}{40}$ & 0 & 0 & $\frac{1}{40}$ & & & & $\frac{81}{140}$ & 7 \\
\hline \multirow[b]{2}{*}{4} & 9 & $\frac{116}{2835}$ & $\frac{-512}{2835}$ & 0 & $\frac{512}{2835}$ & $\frac{-116}{2835}$ & & & $\frac{10240}{693}$ & 11 \\
\hline & 7 & $\frac{20}{315}$ & 0 & 0 & 0 & $\frac{-20}{3155}$ & & & $\frac{2048}{315}$ & 9 \\
\hline \multirow[b]{2}{*}{5} & 11 & $\frac{36975}{1064448}$ & $\frac{-314375}{1064448}$ & $\frac{-272500}{1064448}$ & $\frac{272500}{1064448}$ & $\frac{314375}{1064448}$ & $\frac{-369975}{1064448}$ & & $\frac{3610625}{12012}$ & 13 \\
\hline & 9 & $\frac{1725}{36288}$ & $\frac{-3750}{36288}$ & 0 & 0 & $\frac{3750}{36288}$ & $\frac{-1725}{36288}$ & & $\frac{68125}{924}$ & 11 \\
\hline \multirow[b]{2}{*}{6} & 13 & $\frac{30711}{10010000}$ & $\frac{-409536}{10010000}$ & $\frac{-726975}{10010000}$ & 0 & $\frac{726975}{1001 \cdot 10^{4}}$ & $\frac{409536}{1001 \cdot 10^{4}}$ & $\frac{-30711}{1001 \cdot 10^{4}}$ & $\frac{1306368}{143}$ & 15 \\
\hline & 11 & $\frac{111}{2750}$ & $\frac{-486}{2750}$ & 0 & 0 & 0 & $\frac{486}{2750}$ & $\frac{-111}{2750}$ & $\frac{8374752}{5005}$ & 13 \\
\hline
\end{tabular}

\section{Numerical Results}

The MATLAB locally adaptive (the subintervals are processed from left to right until the integral over each subinterval satisfies the relative error requirement) integration procedures: $n c g 9$ and hermit5 were developed. Procedure $n c g 9$ uses the closed nine-point Newton-Cotes quadrature formula. Procedure 
Table 5. The coefficients of the truncation error formula of Hermitian quadrature.

\begin{tabular}{|c|c|c|c|c|c|c|c|}
\hline$n \delta$ & $w_{0}$ & $w_{1}$ & $w_{2}$ & $w_{3}$ & $w_{4}$ & $w_{5}$ & $w_{6}$ \\
\hline $1 \delta_{1}^{1}$ & $\frac{1}{6}$ & $\frac{-1}{6}$ & & & & & \\
\hline $2 \delta_{2}^{1}$ & $\frac{1}{5}$ & 0 & $\frac{-1}{5}$ & & & & \\
\hline $3 \delta_{3}^{2}$ & $\frac{-81}{1120}$ & $\frac{81}{1120}$ & $\frac{81}{1120}$ & $\frac{-81}{1120}$ & & & \\
\hline $4 \delta_{4}^{2}$ & $\frac{-704}{8505}$ & $\frac{-1024}{8505}$ & $\frac{3456}{8505}$ & $\frac{-1024}{8505}$ & $\frac{-704}{8505}$ & & \\
\hline $5 \delta_{5}^{2}$ & $\frac{-1021875}{19180064}$ & $\frac{-5518125}{19180064}$ & $\frac{6540000}{19180064}$ & $\frac{6540000}{19180064}$ & $\frac{-5518125}{19180064}$ & $\frac{-1021875}{19180064}$ & \\
\hline $6 \delta_{6}^{2}$ & $\frac{-442647}{10010000}$ & $\frac{-4807728}{10010000}$ & $\frac{-1211625}{10010000}$ & $\frac{12924000}{10010000}$ & $\frac{-1211625}{10010000}$ & $\frac{-4807728}{10010000}$ & $\frac{-442647}{10010000}$ \\
\hline
\end{tabular}

Table 6. The coefficients of the truncation error formula of Hermitian quadrature.

\begin{tabular}{ccccccccc}
\hline$n$ & $\delta$ & $w_{0}^{*}$ & $w_{1}^{*}$ & $w_{2}^{*}$ & $w_{3}^{*}$ & $w_{4}^{*}$ & $w_{5}^{*}$ & $w_{6}^{*}$ \\
\hline 1 & $\delta_{1}^{1}$ & $\frac{1}{12}$ & $\frac{1}{12}$ & & & & & \\
2 & $\delta_{2}^{1}$ & $\frac{1}{15}$ & $\frac{4}{15}$ & $\frac{1}{15}$ & & & & \\
3 & $\delta_{3}^{2}$ & $\frac{-27}{1120}$ & $\frac{-81}{1120}$ & $\frac{81}{1120}$ & $\frac{27}{1120}$ & & & \\
4 & $\delta_{4}^{2}$ & $\frac{-64}{2835}$ & $\frac{-512}{2835}$ & 0 & $\frac{512}{2835}$ & $\frac{64}{2835}$ & & \\
5 & $\delta_{5}^{2}$ & $\frac{-40875}{3193344}$ & $\frac{-613125}{3193344}$ & $\frac{-817500}{3193344}$ & $\frac{613125}{3193344}$ & $\frac{40875}{3193344}$ & & \\
6 & $\delta_{6}^{2}$ & $\frac{-9693}{10010000}$ & $\frac{-232632}{10010000}$ & $\frac{-726975}{10010000}$ & 0 & $\frac{726975}{10010000}$ & $\frac{232632}{10010000}$ & $\frac{-9693}{10010000}$ \\
\hline
\end{tabular}

hermit 5 uses the closed five-point Hermite quadrature formula. Both formulas have degree of precision equal to nine. Formulas (2.3) and (2.4) are used for local error estimation in procedures ncg 9 and hermit 5 respectively.

Rounding error may influence both the estimate of the local integral and the evaluation of truncation error. We will, as in [3], define a certain noise level for the problem. All local errors below this noise level in both procedures $n c g 9$ and hermit5 are defined to be zero, thus avoiding subdividing such intervals further. The noise level in both procedures is: $i s * 10^{-17}$, where $i s \neq 0$, is a rough estimate of a modulus of the integral.

In both procedures we are using the conventional termination criteria over each subinterval $[u, v]$ of the initial interval $[a, b]$ :

$$
(i s+\delta=i s) \text { or }(m=u) \text { or }(m=\nu),
$$

where $m=(u+\nu) / 2$ and $\delta$ is the truncation error value over $[u, v]$.

In comparing adaptive quadrature routines, the most important characteristics are [9]:

- efficiency, as measured by the number of function evaluations required to meet a given error tolerance;

- reliability, the extent to which the requested error tolerance is achieved; 
- tolerance responsiveness, as measured by the efficiency of sensitiveness to changes in the error tolerance.

We have tested both procedures ncg 9 and hermit 5 discussed in this paper on 23 test problems used in $[3,9]$. They are picked from two different sources: the 21 first problems come from Kahaner [11] and the last two are picked from [10].

1. $\int_{0}^{1} \exp (x) d x . \quad 2 . \int_{0}^{1} f(x) d x$, where $f(x)=\left\{\begin{array}{l}1, \text { if } x>0.3, \\ 0, \text { else. }\end{array}\right.$

3. $\int_{0}^{1} \sqrt{x} d x . \quad 4 . \int_{-1}^{1}\left(\frac{23}{25} \cosh (x)-\cos (x)\right) d x . \quad 5 . \int_{0}^{1} \frac{d x}{x^{4}+x^{2}+0.9}$.

6. $\int_{0}^{1} \sqrt{x^{3}} d x . \quad 7 . \int_{0}^{1} \frac{1}{\sqrt{x}} d x . \quad 8 . \int_{0}^{1} \frac{1}{x^{4}} d x . \quad 9 . \int_{0}^{1} \frac{2}{2+\sin (10 \pi x)} d x$.

10. $\int_{0}^{1} \frac{1}{1+x} d x . \quad 11 . \int_{0}^{1} \frac{1}{1+e^{x}} d x . \quad 12 . \int_{0}^{1} \frac{x}{e^{x}-1} d x$.

13. $\int_{0,1}^{1} \sin \left(\frac{100 \pi x}{\pi x}\right) d x . \quad 14 . \int_{0}^{10} \frac{\sqrt{50}}{\exp \left(50 \pi x^{2}\right)} d x . \quad 15 . \int_{0}^{10} \frac{25}{\exp (25 x)} d x$.

16. $\int_{0}^{10} \frac{50}{\pi\left(2500 x^{2}+1\right)} d x . \quad 17 . \int_{0,01}^{1} \frac{50 \sin (50 \pi x)}{(50 \pi x)^{2}} d x$.

18. $\int_{0}^{10} \cos (\cos (x)+3 \sin (x)+2 \cos (2 x)+3 \sin (2 x)+3 \cos (3 x)) d x$.

19. $\int_{0}^{1} f(x) d x, \quad f(x)=\left\{\begin{array}{l}\log x, \text { if } x>10^{-15}, \\ 0, \text { else. }\end{array} \int_{-1}^{1} \frac{1}{1.005+x^{2}} d x\right.$.

21. $\int_{0}^{1} \sum_{i=1}^{3} \frac{1}{\cosh \left(20^{i}(x-21)\right)} d x .22 . \int_{0}^{1} 4 \pi^{2} x \sin (20 \pi x) \cos ((2 \pi x)) d x$.

23. $\int_{0}^{1} \frac{1}{1+(230 x-30)^{2}} d x$.

We compared our results with results of MATLAB 7 function quadl [9] and with results of procedure coteda [3]. The procedures quadl and coteda as ncg9 and hermit5 have degree of precision nine. The function quadl uses 
the same stopping criterion (1.5) and is based on a four-point Gauss-Lobatto rule with a three-point Kronrod extension. The second Kronrod extension of applied to the initial interval $[a, b]$ provides the estimate of is in formula (1.5) (procedures $n c g 9$ and hermit 5 also use this formula for rough estimate of the modulus of integral).

The procedure coteda uses both the five-point closed Newton-Cotes rule and the closed nine-point Newton-Cotes rule in a locally doubly adaptive fashion based on bisection. The code is allowed to stop either because the five-point estimate is considered good enough or because the nine-point estimate is considered good enough. The nine-point estimate requires four new points to the five-point estimate, but these are the same points needed in two applications of five-point rule after bisection of the the interval [3]. The procedure coteda uses a sequence of null rules of decreasing degrees [2].

We have tested both new procedures ncg9 and hermit5, as in [3] for twelve different error tolerances $\varepsilon=10^{-1}, 10^{-2} \ldots, 10^{-12}$. Some results of calculations are presented in the following tables. Here we report the number of function evaluations used by each of the four codes. The results of column coteda are taken from [3] and all results of the other columns are calculated. A minus sign in front of number indicates that the requested accuracy was not achieved by the given code in this particular case.

Table 7. Test problem 3 .

\begin{tabular}{lcccc}
\hline$\varepsilon$ & ncg9 & herm5 & quadl & coteda \\
\hline $10^{-1}$ & 9 & 10 & 18 & 9 \\
$10^{-2}$ & 9 & 34 & 18 & 21 \\
$10^{-4}$ & 41 & 66 & 48 & 41 \\
$10^{-6}$ & 73 & 106 & 78 & 73 \\
$10^{-8}$ & 121 & 162 & 168 & 113 \\
$10^{-10}$ & 185 & 242 & 348 & 193 \\
$10^{-11}$ & 265 & 330 & 438 & 241 \\
$10^{-12}$ & 329 & 410 & 588 & 297 \\
\hline
\end{tabular}

Table 9. Test problem 13 .

\begin{tabular}{lcccc}
\hline$\varepsilon$ & ncg 9 & herm5 & quadl & coteda \\
\hline $10^{-1}$ & -17 & 250 & -18 & 513 \\
$10^{-2}$ & 257 & 306 & -78 & 513 \\
$10^{-4}$ & 505 & 530 & 738 & 961 \\
$10^{-6}$ & 969 & 1018 & 1218 & 1089 \\
$10^{-8}$ & 1233 & 1938 & 3138 & 2017 \\
$10^{-10}$ & 2041 & 2962 & 5718 & 3273 \\
$10^{-11}$ & 3129 & 3970 & 6978 & 3977 \\
$10^{-12}$ & 4025 & 4298 & -10068 & 4081 \\
\hline
\end{tabular}

Table 8. Test problem 4 .

\begin{tabular}{lcccc}
\hline$\varepsilon$ & ncg9 & herm5 & quadl & coteda \\
\hline $10^{-1}$ & 9 & 10 & 18 & 9 \\
$10^{-2}$ & 9 & 10 & 18 & 9 \\
$10^{-4}$ & 9 & 10 & 18 & 9 \\
$10^{-6}$ & 9 & 10 & 18 & 9 \\
$10^{-8}$ & 17 & 18 & 18 & 17 \\
$10^{-10}$ & 33 & 34 & 48 & 33 \\
$10^{-11}$ & 33 & 50 & 48 & 33 \\
$10^{-12}$ & 49 & 50 & 48 & 65 \\
\hline
\end{tabular}

Table 10. Test problem 17.

\begin{tabular}{lcccc}
\hline$\varepsilon$ & ncg9 & herm5 & quadl & coteda \\
\hline $10^{-1}$ & 33 & 66 & 18 & 41 \\
$10^{-2}$ & 65 & 146 & -48 & 265 \\
$10^{-4}$ & -193 & 362 & -138 & -297 \\
$10^{-6}$ & -321 & 674 & 618 & 905 \\
$10^{-8}$ & 1025 & 954 & 1068 & 1185 \\
$10^{-10}$ & 1529 & 2090 & 1998 & 2017 \\
$10^{-11}$ & 2162 & 2506 & 2958 & 2273 \\
$10^{-12}$ & 2537 & 3458 & 3888 & 2849 \\
\hline
\end{tabular}


Table 11. Test problem 21.

\begin{tabular}{lcccc}
\hline$\varepsilon$ & ncg9 & herm5 & quadl & coteda \\
\hline $10^{-1}$ & 33 & 18 & 18 & 49 \\
$10^{-2}$ & 48 & -18 & 48 & 77 \\
$10^{-3}$ & 73 & 74 & 78 & -93 \\
$10^{-4}$ & -81 & 106 & 168 & -129 \\
$10^{-5}$ & -105 & -106 & -228 & -161 \\
$10^{-6}$ & -129 & -186 & -228 & -193 \\
$10^{-7}$ & -185 & -226 & -348 & 381 \\
$10^{-8}$ & -233 & -306 & -438 & 461 \\
$10^{-9}$ & 465 & -402 & 888 & 581 \\
$10^{-10}$ & 577 & -434 & 1248 & 737 \\
$10^{-11}$ & 729 & 946 & 1608 & 913 \\
$10^{-12}$ & 953 & 1146 & 2268 & 1117 \\
\hline
\end{tabular}

Table 12. Test problem 22 .

\begin{tabular}{lcccc}
\hline$\varepsilon$ & ncg9 & herm5 & quadl & coteda \\
\hline $10^{-1}$ & 65 & 90 & 168 & 129 \\
$10^{-2}$ & 105 & 122 & 168 & 129 \\
$10^{-3}$ & 121 & 136 & 228 & 177 \\
$10^{-4}$ & 145 & 242 & 408 & 257 \\
$10^{-5}$ & 257 & 258 & 648 & 257 \\
$10^{-6}$ & 257 & 354 & 798 & 289 \\
$10^{-7}$ & 369 & 498 & 1038 & 465 \\
$10^{-8}$ & 505 & 506 & 1248 & 505 \\
$10^{-9}$ & 505 & 842 & 2208 & 529 \\
$10^{-10}$ & 873 & 1002 & 3018 & 833 \\
$10^{-11}$ & 1009 & 1122 & 3978 & 985 \\
$10^{-12}$ & 1169 & 1866 & 5418 & 1033 \\
\hline
\end{tabular}

The following observations can be made.

1. The results $n c g 9$ and coteda are very similar in both reliability and efficiency. It can be explained that the code $n c g 8$ uses the closed nine-point Newton-Cotes formula for integral approximation and the same strong 7 -th degree of exactness null rule for locally error evaluation. The code coteda, as it was already mentioned, uses both closed five-point and nine-point NewtonCotes formulas for integral approximation and a sequence of null rules of decreasing degrees for locally error evaluation. The code coteda in contrast to code $n c g 9$ uses the locally doubly adaptive strategy [3]. It is known that usage of the the null rules of lower degrees usually requires computation of larger number of integrand functions. But a doubly adaptive strategy usually requires computation of less values of integrand function than a single adaptive strategy. Therefore only in 99 cases from all $23 \times 12=276$ cases code $n c g 9$ required a smaller number of computations of integrand functions than code coteda.

2. Only for four tests: test 3 (the singular problem) for tolerance $10^{-1}$, test 13 (the nonlinear oscillatory problem) for tolerance $10^{-1}$, test 17 (the nonlinear oscillatory problem) for tolerance $10^{-6}$, and test 21 (three peaks problem; peak in the vicinity $x=0.6$ is very narrow) for tolerances $10^{-7}$ and $10^{-8}$ code coteda gives better results than $n c g 9$. It can be explained that for this difficult tests the 7-th degree of exactness null rule is too "optimistic". The error estimation procedure based on sequence of null rules of decreasing degrees presents more exact results for these cases.

3. The codes hermit5 and quadl based on the nine degree Hermite and Gauss-Lobatto rule with a three-point Kronrod extension respectively in all cases demonstrate worse results in efficiency than codes $n c g 9$ and coteda. It can be explained that in the procedures hermit5 and quadl integration step is larger than in codes ncg 9 and coteda. 
4. In terms of reliability the procedures ncg9, hermit5 and coteda demonstrate very close results. The reliability of code quadl is slightly worse than reliability of other discussed procedures.

\section{Conclusions}

In automatic quadrature algorithms the estimate of the truncation error is a very important step: it governs the decision on whether to return the current approximation and terminate or to continue calculations. Both the efficiency and the reliability therefore depend heavily on the error estimating procedure. Consequently the coefficients of the highest degree null rules with the smallest errors based on the same set of the points for $3,5,7,9$, and 11 point NewtonCotes quadrature formulas are calculated in this paper. The coefficients of the $2,3,4,5,6$, and 7 point Hermite quadrature formulas and the coefficients of their error estimation formulas are calculated. The calculated Hermitian error estimation formulas have the highest degree and the smallest error also.

In view of these (admittedly limited) test results we can conclude that both codes cteda and $n c g 9$ show the best and very closed results in both reliability and efficiency. We can conclude:

1) The closed nine-point Newton-Cotes formula puts up better performance than the same degree Gauss-Lobatto formula;

2) Reliability of both error estimation formulas (based on one null rule and on a sequence null rules) are the same for the tolerances $\leq 10^{-4}$;

3) For the tolerances $\geq 10^{-3}$ the error estimation formula of procedure $n c g 9$ gives less reliable results than the error estimation formula of code cteda. Therefore the error estimation formulas, presented in this paper, can be used for approximation of definite integrals, in particular, if the required tolerance $\leq 10^{-4}$. Efficiency of code cteda can be explained that it uses double adaptive integration procedure.

The procedure hermit5 shows results of reliability better than results of the same degree procedure $n c g 9$. But efficiency of code hermit 5 is worse than of procedure $n c g 9$. Therefore, Hermitian quadrature can be used for approximated of integrals if calculation of the values of derivates of integrand function is not very complicated.

\section{References}

[1] N.S. Bakhvalov. Numerical Methods, volume 1. Moscow. (In Russian)

[2] J. Berntsen and T.O. Espelid. Error estimation in automatic quadrature routines. ACM Transactions on Mathematical Software (TOMS), 17(2), 233-252, 1991.

[3] T.O. Espelid. Doubly adaptive quadrature routines based on Newton-Cotes rules. BIT Numerical Mathematics, 43, 319-337, 2003. 
[4] T.O. Espelid. A test of QUADPACK and four double adaptive quadrature routines. Reports in Informatics, 281, 1-22, 2004.

[5] T.O. Espelid and T. Sorevik. A discussion of a new error estimate for adaptive quadrature. BIT Numerical Mathematics, 29(2), 283-294, 1989.

[6] P. Favati, G. Fiorentino, G. Lotti and F. Romani. Local error estimates and regularity tests for the implementation of double adaptive quadrature. $A C M$ Transactions on Mathematical Software (TOMS), 23(1), 16-31, 1997.

[7] P. Favati, G. Lotti and F. Romani. Algorithm 691; improving QUADPACK automatic integration routines. ACM Transactions on Mathematical Software (TOMS), 17(2), 218-232, 1991.

[8] P. Favati, G. Lotti and F. Romani. Interpolatory integration formulas for optimal composition. ACM Transactions on Mathematical Software (TOMS), $\mathbf{1 7}(2), 207-217,1991$.

[9] W. Gander and W. Gautschi. Adaptive quadrature-revisited. BIT, 40, 84-101, 2000.

[10] S. Gariba, L. Quartapelle and G. Reina. Algorithm 36-SNIFF: Efficient selftuning algorithm for numerical integration. Computing, 20, 363-375, 1978.

[11] D.K. Kahaner. Comparison of numerical quadrature formulas. In: J. Rice(Ed.), Mathematical Software, New-York, Academic Press, 229-259, 1971.

[12] B. Kvedaras and M. Sapagovas. Numerical methods. Mintis, Vilnius, 1974. (In Lithuanian)

[13] D.P. Laurie. Sharper error estimates in adaptive quadrature. BIT, 23, 258-261, 1983.

[14] D.P. Laurie. Anti-Gaussian quadrature formulas. Mathematics of Computation, 65(214), 739-747, 1996.

[15] J.N. Lyness. Symmetric integration rules for hypercubes III. construction of integration rules using null rules. Math. Comput., 19, 625-637, 1965.

[16] W. M. McKeeman. Certification of algorithm 145. adaptive numerical integration by Simpson's rule. Commun. ACM, 6, 167-168, 1963.

[17] Ch. W. Ueberhuber. Numerical Computation 2: Methods, Software, and Analysis. Springer-Verlag Berlin Heidelberg New York, 1997. 